Pacific Journal of Mathematics

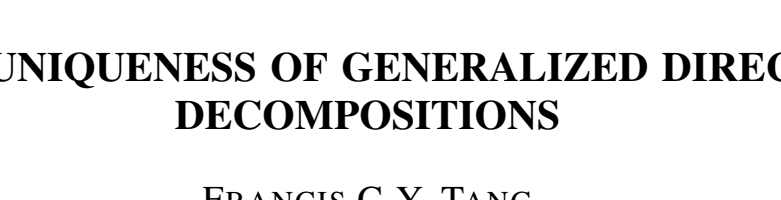




\title{
ON UNIQUENESS OF GENERALIZED DIRECT DECOMPOSITIONS
}

\author{
C. Y. TANG
}

Generalized direct products were introduced by B. H. Neuman and $H$. Neumann. In this paper we attempt to study some properties of generalized direct decompositions of groups. In general, decompositions of a given group into indecomposable generalized direct factors are not unique up to isomorphisms. The main result of this paper is that if the commutator subgroup of $G$ is a cyclic $p$-group contained in the center $Z(G)$ then any two generalized direct decompositions of $G$ into indecomposable generalized direct factors with respect to its center $Z(G)$ of $G$ are isomorphic modulo $Z(G)$.

Generalized direct products with amalgamated subgroups were defined externally by B. H. Neumann and H. Neumann in [1]. Different existence theorems of such products for given amalgams of groups were given in [1], [2], [3], [4]. The main application so far has been in the construction of groups. It seems to us that it is of interest to study the internal structure of such products. In particular, we like to obtain some information concerning the decomposition of a given group relative to some $H \subset Z(G)$ into factors indecomposable relative to the same $H$. Examples can easily be constructed to show that such decompositions may not necessarily be unique up to isomorphisms. The main difficulty is due to the fact that intersections of the commutator subgroups of the factors may not be trivial. In the following investigation we shall restrict ourselves to the special case when the generalized direct product has only a single amalgamated subgroup.

In $\S 2$ we shall develop some simple properties concerning the exchangeability of factors in different decompositions. In $\S 3$ we obtain a characterization of groups which are generalized directly indecomposable with respect to their centers for the case of nilpotent groups of class two with cyclic commutator subgroups. With this result we are able to show that for a nilpotent group of class two with a finite cyclic commutator subgroup any decompositions into generalized direct indecomposable factors with respect to its center $Z(G)$ are isomorphic $\bmod Z(G)$. It is of interest to note that, applying a theorem of Weichsel [5], for $Z(G)=C(G)$ we can give a characterization of critical $p$-groups in terms of generalized direct indecomposability with respect to the center.

Since it is too difficult to study generalized direct decompositions with respect to more than one amalgamated subgroup we shall only 
attempt to define generalized direct decomposition with respect to a single amalgamated subgroup. Moreover in our investigation we shall always assume the double chain condition of Zassenhaus [6] so that only a finite number of factors is involved.

Definition 1.1. A group $G$ is said to have a generalized direct decomposition with respect to a subgroup $H$ if there exist subgroups $G_{1}, \cdots, G_{n}$ such that,

(i) $G$ is generated by $G_{1}, \cdots, G_{n}$,

(ii) $G_{i}$ and $G_{j}$ commute elementwise for all $i \neq j$,

(iii) $G_{i}$ contains $H$ for all $i$,

(iv) the intersection of $G_{i}$ with the subgroup generated by

$$
G_{1}, \cdots, G_{i-1}, G_{i+1}, \cdots, G_{n}
$$

is exactly $H$ for all $i$.

$G_{i}$ will be called a generalized direct factor of $G$ with respect to $H$, and we shall denote such a decomposition by $G=\left(G_{1} \times \cdots \times G_{n}\right)_{H}$. If there does not exist such a set of subgroups other than $G$ and $H$ then we shall say that $G$ is generalized directly indecomposable with respect to $H$.

It is to be noted that $H$ must be contained in the center of $G$.

Notations AND Terminology. For abbreviation we shall call a generalized direct decomposition with respect to $H$ an $H$-decomposition. Correspondingly we shall also use the terms $H$-products, $H$ factors and $H$-indecomposable.

We shall adopt the following notations:

$$
\begin{aligned}
\prod_{i=1}^{n} G_{i} & =G_{1} \times \cdots \times G_{n} \\
\left(\prod_{i=1}^{n} G_{i}\right)_{H} & =\left(G_{1} \times \cdots \times G_{n}\right)_{H}
\end{aligned}
$$

If $G=\prod_{i=1}^{n} G_{i}$ then $G_{i}^{\prime}=\prod_{\nu \neq i}^{n} G_{\nu}$ and in the same way if $G=$ $\left(\prod_{i=1}^{n} G_{i}\right)_{H}$ then $G_{l}^{\prime}=\left(\prod_{\nu \neq i}^{n} G_{\nu}\right)_{H}$.

If $G=\left(\prod_{i=1}^{n} G_{i}\right)_{B}$ then $\bar{G}$ and $\bar{G}_{i}$ will always mean $G / H$ and $G_{i} / H$ respectively. Moreover if $x \in G$ then $\bar{x}$ will be the image of $x$ in $\bar{G}$.

For $G=\prod_{i=1}^{n} G_{i}$ the $G_{i}$-decomposition operator is to be understood in the sense of Zassenhaus [6].

The commutator of the elements $x$ and $y$ is denoted by $[x, y]$.

$[A, B]$ denotes the subgroup generated by the set of all commutators $[x, y]$ where $x \in A$ and $y \in B$.

$\left\{x_{1}, \cdots, x_{n}\right\}$ and $\left\{x_{1}, \cdots, x_{n}, H\right)$ will mean the group generated by $x_{1}, \cdots, x_{n}$ and the group generated by $x_{1}, \cdots, x_{n}$ together with elements of $H$ respectively. 
$Z(G)$ and $C(G)$ will be the center and the commutator subgroup of $G$ respectively.

Definition 1.2. Every $H$-decomposition of $G$ induces a direct decomposition of $\bar{G}$. Thus two $H$-decompositions of $G$ are said to be isomorphic mod $H$ if and only if their induced direct decompositions of $\bar{G}$ are isomorphic.

It is not difficult to establish the following results:

THeOREM 1.3. If $G$ is abelian then any $H$-decompositions into indecomposable $H$-factors are isomorphic $\bmod H$.

THEOREM 1.4. If $G$ splits over $H$ then any $H$-decompositions into indecomposable $H$-factors are isomorphic. Indeed if $Z(G)=H$ then the decomposition is unique.

2. Definition 2.1. Let $G=\left(\prod_{i=1}^{n} G_{i}\right)_{H}=\left(\prod_{i=1}^{m} F_{i}\right)_{H}$ be two $H$ decompositions of $G$. Then the factors $G_{i}$ and $F_{j}$ are said to be exchangeable if $\left(F_{j} \times G_{i}^{\prime}\right)_{H}$ and $\left(G_{i} \times F_{j}^{\prime}\right)_{H}$ are $H$-decompositions of $G$. Two $H$-decompositions of $G$ are said to be exchangeable if each factor of one decomposition is exchangeable with some factor of the other decomposition.

The following lemma is well known.

LEMmA 2.2. Let $G=\prod_{i=1}^{n} G_{i}=\prod_{i=1}^{m} F_{i}$ and let $\theta_{i}$ and $\phi_{i}$ denote the $F_{i}=$ and $G_{i}$-decomposition operators respectively. $G_{i}$ is exchangeable with $F_{j}$ if and only if $\theta_{j}$ and $\phi_{i}$ induce isomorphisms between $G_{i}$ and $F_{j}$.

Lemma 2.3. If $G=G_{1} \times G_{2} \times A=F_{1} \times F_{2} \times B$ where $G_{1}$ and $G_{2}$ are exchangeable with $F_{1}$ and $F_{2}$ respectively, then $\left(G_{1} \times G_{2}\right)$ is exchangeable with $\left(F_{1} \times F_{2}\right)$.

Proof. Since $G_{1}$ and $F_{1}$ are exchangeable we have

$$
G=G_{1} \times G_{2} \times A=G_{1} \times F_{2} \times B .
$$

Let $\theta$ map $G$ onto $G \theta$ with $\operatorname{ker} \theta=G_{1}$. Then

$$
G \theta=G_{2} \theta \times A \theta=F_{2} \theta \times B \theta .
$$

Now $G_{1} \cap G_{2}=G_{1} \cap F_{2}=1$. Therefore, the $G_{2} \theta$ - and $F_{2} \theta$-decomposition operators induce isomorphisms between $G_{2} \theta$ and $F_{2} \theta$. Hence

$$
G \theta=G_{2} \theta \times B \theta=F_{2} \theta \times A \theta .
$$


But this implies that ker $\theta \cap\left(G_{2} \times B\right)=1$. It follows that

$$
G=G_{1} \times G_{2} \times B \text {. }
$$

In the same way we can show that $G=F_{1} \times F_{2} \times A$.

CoRollaRY 2.4. If $G=\prod_{i=1}^{n} G_{i}=\prod_{i=1}^{m} F_{i}$ such that $G_{i}$ and $F_{i}$ are exchangeable for $1 \leqq i \leqq k$, then

$$
\begin{aligned}
G & =F_{1} \times \cdots \times F_{k} \times G_{k+1} \times \cdots \times G_{n} \\
& =G_{1} \times \cdots \times G_{k} \times F_{k+1} \times \cdots \times F_{m} .
\end{aligned}
$$

THEOREM 2.5. If $G=\left(\prod_{i=1}^{n} G_{i}\right)_{H}=\left(\prod_{i=1}^{m} F_{i}\right)_{H}$, where $H=Z(G)$, are two exchangeable $H$-decompositions in which none of the factors is trivial, then the two H-decompositions are identical.

Proof. Let $G_{1}$ and $F_{1}$, say, be two exchangeable factors. If $x \in G_{1}$ then $x=a b$ for some $a \in F_{1}$ and $b \in F_{1}^{\prime}$. But the exchangeability of $G_{1}$ and $F_{1}$ implies that $b \in Z\left(F_{1}^{\prime}\right) \subset Z(G)=H$. Hence $G_{1} \subset F_{1}$. In the same way $F_{1} \subset G_{1}$, whence $F_{1}=G_{1}$. Clearly each $G_{i}$ coincides with one and only one $F_{j}$. Thus applying Lemma 2.4 to $\bar{G}$ we have $m=n$. This proves tne theorem.

Since any two Remak decompositions of a given group are exchangeable the following well-known result becomes an immediate consequence of this theorem.

CoRollary 2.6. If $Z(G)=1$ then $G$ admits a unique remark decomposition.

3. In this section we shall be mainly concerned with nilpotent groups of class two since this is the simplest case after abelian groups. In particular we shall study the $H$-decompositions of $G$ when $H=$ $Z(G)$. $H$-products with $H=Z(G)$ are referred to as central products by P. Hall. Therefore, we shall call $G=\left(\prod_{i=1}^{n} G_{i}\right)_{H}$ a central decomposition of $G$ whenever $H=Z(G)$. It is to be noted that in a central decomposition the center of each factor coincides with the center of the group. Thus in a central decomposition of $G$ into centrally indecomposable factors each factor $G_{i}$ is indeed indecomposable with respect to its center $Z\left(G_{i}\right)$. When $G$ is nilpotent of class two with a cyclic commutator subgroup we are able to give a complete characterization of centrally indecomposable groups.

LemMA 3.1. Let $G=(A \times B)_{H}=(C \times D)_{H}$. If $x$ is any element of $C$ then there exist $a \in A$ and $b \in B$ with $a$ and $b$ respectively of the form $a=c d$ and $b=c^{*} d^{-1}$ where $c, c^{*} \in C$ and $d \in D$ such that 
$x=a b$.

Proof. Let $x \in C$. Then $x=a b$ for some $a \in A$ and $b \in B$. Now $a=c d$ and $b=c^{\prime} d^{\prime}$ for some $c, c^{\prime} \in C$ and $d, d^{\prime} \in D$. Thus $x=c c^{\prime} d d^{\prime}$, whence $d d^{\prime}=h \in C \cap D=H$. Writing $c^{*}=c^{\prime} h \in C$ we have $b=c^{*} d^{-1}$. This proves the lemma.

Lemma 3.2. Let $G=(A \times B)_{H}=(C \times D)_{H}$. If $a \in A$ and $a=c d$ with $c \in C$ and $d \in D$ then $[c, B] \subset H$ and also $[d, B] \subset H$.

Proof. Consider $\bar{G}=\bar{A} \times \bar{B}=\bar{C} \times \bar{D}$. Then $\bar{a}=\bar{c} \bar{d}$ centralizes $\bar{B}$. Since $\bar{C} \cap \bar{D}=1$ this implies that $\bar{c}$ and $\bar{d}$ centralize $\bar{B}$, whence $[c, b]=[b, d] \in H$ for all $b \in B$.

DeFinition 3.3. Let $G=\left(\prod_{i=1}^{n} G_{i}\right)_{H}$ and $\theta$ be the homomorphism mapping $G$ onto $\bar{G}$. Then the $H$-projection of $x \in G$ in $G_{i}$ is defined to be the set of all preimages of $\bar{x} \theta_{i}$ under $\theta$, where $\theta_{i}$ is the $\bar{G}_{i^{-}}$decomposition operator of $\bar{G}=\prod_{i=1}^{n} \bar{G}_{i}$. We shall denote this set by $P_{G_{i}}(x)$.

It is easy to see that the $H$-projection of any subgroup of $G$ in $G_{i}$ is a subgroup of $G_{i}$ containing $H$.

From now on, unless otherwise specified, we shall always take $H=Z(G)$.

Lemma 3.4. Let $G=(A \times B)_{H}=(C \times D)_{H}$. Let $M$ be a subgroup of $A$ containing $H$ such that $M \subset P_{A}(C)$ and $P_{o}(M)=C$. Then $A=$ $(M \times N)_{H}$ where $N=A \cap D$.

Proof. Let $x$ be any element of $A$. Then $x=c d$ for some $c \in C$ and $d \in D$. Since $P_{o}(M)=C$ this implies that there exists $u \in M$ such that $u=c d_{1}, d_{1} \in D$. Therefore $x=u d_{1}^{-1} d$, whence $d_{1}^{-1} d=u^{-1} x \in A \cap D$. Hence $A=\{M, N\}$.

Now $M \subset P_{A}(C)$ implies that $M \subset C B$. But this implies that $[M, N]=1$.

Finally $\{M, N\}=A$ together with $[M, N]=1$ implies that

$$
M \cap N \subset Z(A) \subset Z(G)=H .
$$

Since $H \subset M \cap N$, it follows that $A=(M \times N)_{H}$.

Corollary 3.5. Let $G=(A \times B)_{H}=(C \times D)_{H}$ where $A$ is $H$ indecomposable. If $M$ is a subgroup of $A$ containing $H$ such that $M \subset P_{A}(C)$ and $P_{o}(M)=C$ then $M=A$.

As it was pointed out in $\S 1$ that the main difficulty in the study 
of such decompositions is due to the fact that, in general, $C\left(G_{i}\right)$ and $C\left(G_{i}^{\prime}\right)$ will not intersect trivially. If, however, $C\left(G_{i}\right) \cap C\left(G_{i}^{\prime}\right)=1$ for all $i$ then we have the following strong result.

THEOREM 3.6. If $G=\left(\prod_{i=1}^{n} G_{i}\right)_{H}$ is a central decomposition of $G$ into centrally indecomposable factors such that $C\left(G_{i}\right) \cap C\left(G_{i}^{\prime}\right)=1$ for all $i$, then $G$ admits a unique central decomposition into centrally indecomposable factors.

Proof. Let $G=\left(\prod_{i=1}^{m} F_{i}\right)_{H}$ be a central decomposition of $G$ into centrally indecomposable factors. Thus $\bar{G}=\prod_{i=1}^{n} \bar{G}_{i}=\prod_{i=1}^{m} \bar{F}_{i}$. Let $\theta_{i}$ and $\phi_{i}$ be the $\bar{G}_{i^{-}}$and $\bar{F}_{i}$-decomposition operators of $\bar{G}$ respectively. Since $\bar{G}_{1}=\prod_{i=1}^{m} \bar{G}_{1 i} \phi_{i} \theta_{1}$ and $\overline{P_{F_{i}}\left(G_{1}\right)}=\bar{G}_{1} \phi_{i}$ it follows that

$$
Q_{i}=P_{A_{1}}\left(P_{F_{i}}\left(G_{1}\right)\right)
$$

is the set of all preimages of $\bar{G}_{1} \phi_{i} \theta_{1}$ under $\theta$, where $G \theta=\bar{G}$, and that $G_{1}=\left\{Q_{1}, \cdots, Q_{m}\right\}$.

Now let $x \in P_{F_{i}}\left(G_{1}\right)$ and $y \in P_{F_{j}}\left(G_{1}\right), i \neq j$. Then $x=s u$ and $y=$ $t v$ where $s, t \in G_{1}$ aud $u, v \in G_{1}^{\prime}$. Since $[x, y]=1$ and $C\left(G_{1}\right) \cap C\left(G_{1}^{\prime}\right)=1$ we have $[s, t]=[u, v]=1$. This implies that $\left[Q_{i}, Q_{j}\right]=1$ for $i \neq j$. Moreover, it is clear that

$$
Q_{i} \cap\left\{Q_{1}, \cdots, Q_{i-1}, Q_{i+1}, \cdots, Q_{m}\right\} \subset Z\left(G_{1}\right) \subset H .
$$

Hence $G_{1}=\left(\prod_{1=1}^{m} Q_{i}\right)_{H}$. But $G_{1}$ is centrally indecomposable. Therefore there exists $i=1$, say, such that $G_{1}=Q_{1}$ and $Q_{i}=H$ for $i \neq 1$. Applying Lemma 3.4 and putting $M=P_{F_{1}}\left(G_{1}\right), A=F_{1}$ and $C=G_{1}$ we have $F_{1}=\left(P_{F_{1}}\left(G_{1}\right) \times\left(F_{1} \cap G_{1}^{\prime}\right)\right)_{H}$, whence $F_{1}=P_{F_{1}}\left(G_{1}\right)$. But

$$
G_{1}=Q_{1}=P_{G_{1}}\left(P_{F_{1}}\left(G_{1}\right)\right)
$$

implies that $G_{1}=P_{G_{1}}\left(F_{1}\right)$. Thus $F_{1}=P_{F_{1}}\left(P_{G_{1}}\left(F_{1}\right)\right)$ and $P_{F_{1}}\left(P_{G_{i}}\left(F_{1}\right)\right)=H$ for all $i \neq 1$.

Let $x \in F_{1}$ and $y \in F_{1}^{\prime}$. Then $x=g_{1} u$ and $y=g_{1}^{*} v$ where $g_{1}, g_{1}^{*} \in G_{1}$ and $u, v \in G_{1}^{\prime}$. Since $P_{G_{1}}\left(F_{1}\right)=G_{1}$ and $C\left(G_{1}\right) \cap C\left(G_{1}^{\prime}\right)=1$, it follows that $g_{1}^{*} \in Z(G)=H$. Therefore $F_{1}^{\prime} \subset G_{1}^{\prime}$. Also $P_{F_{1}}\left(P_{G_{i}}\left(F_{1}\right)\right)=H$ for all $i \neq 1$ implies that $P_{G_{1}}\left(F_{1}\right) \subset F_{1}^{\prime}$ for all $i \neq 1$, whence $u \in Z(G)$. Therefore $F_{1} \subset G_{1}$ and by Corollary $3.5 F_{1}=G_{1}$. This implies that

$$
G=\left(G_{1} \times G_{1}^{\prime}\right)_{H}=\left(G_{1} \times F_{1}^{\prime}\right)_{H} .
$$

Thus $F_{1}^{\prime} \subset G_{1}^{\prime}$ implies that $F_{1}^{\prime}=G_{1}^{\prime}$. Hence by induction $m=n$ and with proper reindexing $F_{i}=G_{i}$ for all $i$.

LEMMA 3.7. The congruences $x+y \equiv 1 \bmod n$ and $x y \equiv 0 \bmod n$ have solutions in $x, y$ other than 0 and $1 \bmod n$ if and only if $n \neq p^{\alpha}$ 
or $\infty$ where $p$ is a prime.

Proof. We shall first show that if $n=p^{\alpha}$ or $\infty$ then the only solutions are 0 and $1 \bmod n$. Let $n=\infty$. Then $x y=0$ implies $x=0$, say, whence $y=1$. On the other hand if $\mathrm{n}=p^{\alpha}$ then $x y \equiv 0 \bmod n$ and $x+y \equiv 1$ mod $n$ imply that $p^{\alpha} \mid x(x-1)$. Thus $p^{\alpha} \mid x$ or $p^{\alpha} \mid$ $(x-1) \equiv-y$. Hence the only solutions are 0 and $1 \bmod n$.

Suppose now $n \neq p^{\alpha}$ or $\infty$. Then there exist integers $\beta, \gamma \neq 1$ such that $n=\beta \gamma$ and $(\beta, \gamma)=1$. This implies that there exists integers $s$ and $t$ such that $s \beta+t \gamma=1$. Let $x=s \beta$ and $y=t \gamma=(1-x)$. Clearly $\beta \mid x$ and $\gamma \mid(x-1)$. On the other hand $n \nmid x$ or $(x-1)$. For, if not, say, $n \mid x=s \beta$. This implies that $\gamma \mid s$, whence $\gamma \mid s \beta+t \gamma=1$. Thus $s \beta$ and ty are solutions to the given congruences with neither of them congruent to $0 \bmod n$.

THEOREM 3.8. Let $G / H$ be an abelian group of rank two. $G$ is centrally indecomposable if and only if $C(G)$ is a cyclic p-group or an infinite cyclic group.

Proof. Since $G / H$ is an abelian group of rank two it implies that there exist $a, b \in G$ such that $G=\{a, b, H\}$ where $a, b \notin H$ and $1 \neq$ $[a, b] \in H$. Let $[a, b]=h$ and ord $h=n$ where $n=p^{k}$ or $\infty$. It is clear that $C(G)=\{h\}$ and the orders of $\alpha$ and $b$ must be either divisble by $n$ or infinite. Indeed if $n=p^{k}$ then $a^{p^{k}}$ and $b^{p^{k}}$ are elements of $H$. Let $G=(A \times B)_{H}$. Then $a=x y$ and $b=x^{\prime} y^{\prime}$ where $x, x^{\prime} \in A$ and $y, y^{\prime} \in B$. But $x=a^{\alpha} b^{\beta} u, y=a^{\gamma} b^{\delta} v, x^{\prime}=a^{\alpha^{\prime}} b^{\beta^{\prime}} u^{\prime}$ and $y^{\prime}=a^{\gamma^{\prime}} b^{\delta^{\prime}} v^{\prime}$ where $u, v, u^{\prime}, v^{\prime} \in H$. Since $[x, y]=1$ we have $\alpha \delta-\beta \gamma \equiv 0 \bmod n$. Moreover $a=x y=a^{\alpha+\gamma} b^{\beta+\delta} h^{-\beta \gamma} u v$ implies $a^{\alpha+\gamma-1} b^{\beta+\delta} h^{-\beta \delta} u v=1$. This This means $a^{\alpha+\gamma-1} b^{\beta+\delta} \in H$. But

$$
\left[a, a^{\alpha+\gamma-1} b^{\beta+\delta}\right]=1
$$

implies $\beta+\delta \equiv 0 \bmod n$ and $\left[b, a^{\alpha+\gamma-1} b^{\beta+\delta}\right]=1$ implies $\alpha+\gamma-1 \equiv 0 \bmod n$ or $\alpha+\gamma \equiv 1 \bmod n$ Hence $0 \equiv \alpha \delta-\beta \gamma \equiv \delta(\alpha+\gamma) \equiv \delta \bmod n$. Applying the same argument to $x^{\prime}$ and $y^{\prime}$ we have $\beta^{\prime}=\delta^{\prime}=1 \bmod n$ and $\alpha^{\prime} \equiv$ $-\gamma^{\prime} \equiv 0 \bmod n$. But $\beta \equiv \delta \equiv \alpha^{\prime} \equiv \gamma^{\prime} \equiv 0 \bmod n \operatorname{implies} b^{\beta}, b^{\delta}, a^{\alpha^{\prime}}$ and $a^{\gamma^{\prime}}$ are elements of $H$. Thus for $\left[x, y^{\prime}\right]=\left[x^{\prime}, y\right]=1$ we must have $\alpha \delta^{\prime} \equiv 0$ and $\beta^{\prime} \gamma \equiv 0 \bmod n$. It follows that $\left(\alpha \beta^{\prime}\right)\left(\gamma \delta^{\prime}\right) \equiv 0 \bmod n$. But $(\alpha+\gamma)\left(\beta^{\prime}+\delta^{\prime}\right) \equiv 1 \bmod n$ implies $\alpha \beta^{\prime}+\gamma \delta^{\prime} \equiv 1 \bmod n$. Hence, by lemma 3.7, the congruences cannot have solutions other than 0 and $1 \bmod n$. Suppose $\alpha \beta^{\prime} \equiv 0$ and $\gamma \delta^{\prime} \equiv 1 \bmod n$. Since $\gamma \equiv 1-\alpha$ and $\delta^{\prime} \equiv 1-\beta^{\prime} \bmod n$ we have $(1-\alpha)\left(1-\beta^{\prime}\right) \equiv 1 \bmod n$ which implies $\beta^{\prime} \equiv-\alpha \bmod n$. Recalling the facts that $\left[x, y^{\prime}\right]=\left[x^{\prime}, y\right]=1$ and $a^{p^{k}}$, $b^{p^{k}} \in H$ we must have $\alpha(1+\alpha) \equiv 0$ and $-\alpha(1-\alpha) \equiv 0 \bmod n$. This 
means $2 \alpha \equiv 0 \bmod n$. If $p \neq 2$ then $\alpha \equiv 0$, which will imply $a, b \in B$. If $p=2$ then $2 \alpha \equiv 0 \bmod 2^{k}$. Thus $\alpha \equiv 0$ or $t 2^{k-1}$ for some odd $t$. Clearly both $\left(1-t 2^{k-1}\right)$ and $\left(1+t 2^{k-1}\right)$ are relatively prime to $2^{k}$ for $k \neq 1$. Since $y=a^{1-\alpha} z$ and $y^{\prime}=b^{1+\alpha} z^{*}$ for some $z, z^{*} \in H$ we have $a, b \in B$. Thus $B=G$. If $k=1$ and $\alpha \equiv 1$ then $G=A$. Similarly, if $\alpha \beta^{\prime}=1$ and $\gamma \delta^{\prime} \equiv 0 \bmod n$ we have $G=A$ or $B$. Hence $G$ is centrally indecomposable.

To prove the converse we shall show that if the order of $C(G)$ is not a prime power or infinity then $G$ is centrally decomposable. Again let $G=\{a, b, H\}$ and $[a, b]=h$ with ord $h=n \neq p^{k}$ or $\infty$. By Lemma 3.7 there exist solutions other than 0 and $1 \bmod n$ to the congruences $x+y=1$ and $x y \equiv 0 \bmod n$. Let $\alpha, \beta$ be such a pair solutions. Let $A=\left\{a^{\alpha}, b^{\alpha}, H\right\}$ and $B=\left\{a^{\beta}, b^{\beta}, H\right\}$. A simple check will show $G=(A \times B)_{H}$.

THEOREM 3.9. Let $G$ be a nilpotent group of class two with a cyclic commutator subgroup. If $G=\left(\prod_{i=1}^{n} G_{i}\right)_{H}$ is a central decomposition of $G$ into centrally indecomposable factors then $\bar{G}_{i}=G_{i} / H$ is an abelian group of rank two.

Proof. Clearly $\bar{G}_{i}$ cannot be of rank one since this will imply $G_{i} \subset Z(G)=H$. Hence $G_{i}$ will not be a proper central factor. We shall therefore assume that $\bar{G}_{i}$ is of rank $r>2$. Thus

$$
G_{i}=\left\{\alpha_{1}, \cdots, \alpha_{r}, H\right\} \text {. }
$$

Since $C(G)$ is cyclic we shall let $C(G)=\{h\}$ where $h \in H$. This implies $C\left(G_{i}\right)=\left\{h^{\nu}\right\}$. Therefore there exist $a, b \in G_{i}$ such that $[a, b]=h^{\nu}=c$. Let $A=\{a, b, H\}$. Clearly $A \neq G_{i}$ since $A / H$ is of rank two. Let $\beta_{1}, \cdots, \beta_{k} \in G_{i}$ such that $G_{i}=\left\{a, b, \beta_{1}, \cdots, \beta_{k}, H\right\}$ and that $k$ is minimal. Let $\left[a, \beta_{i}\right]=c^{\lambda_{i}}$ and $\left[b, \beta_{i}\right]=c^{\mu_{i}}$. Define $B=\left\{\beta_{i} a^{\mu_{i}} b^{-\lambda_{i}}, H ; i=1, \cdots, k\right\}$. Clearly $G_{i}=\{A, B\}$. Also $\left[a, \beta_{i} a_{i}^{\mu_{i}} b^{-\lambda_{i}}\right]=c^{\lambda_{i}} \cdot c^{-\lambda_{i}}=1$ and $\left[b, \beta_{i} a_{i}^{\mu_{i}} b^{-\lambda_{i}}\right]=$ $c^{\mu_{i}} \cdot c^{-\mu_{i}}=1$. Therefore $[A, B]=1$. Furthermore if $x \in A \cap B$ then

$$
x \in Z\left(G_{i}\right) \subset H .
$$

In fact this also says that $A$ cannot be contained in $B$. Hence $G_{i}=$ $(A \times B)_{H}$ contradicting the hypothesis that $G_{i}$ is centrally indecomposable.

Applying Theorems 3.8 and 3.9 the following characterization of centrally indecomposable nilpotent groups of class two with cyclic commutator subgroups is immediate.

THEOREM 3.10. Let $G$ be a nilpotent group of class two with a cyclic commutator subgroup. $G$ is centrally indecomposable if and 
only if $G / H$ is of rank two and $C(G)$ is either a p-group or an infinite group.

CoROLlaRY 3.11. Let $G$ be a nilpotent group of class two with a cyclic center. $G$ is centrally indecomposable if and only if $G / H$ is of rank two and $C(G)$ is either a p-group or an infinite group.

It is of interest to note at this point that applying a theorem of Weichsel [5] we have a characterization of critical p-groups when their centers coincide with their commutator subgroups.

THEOREM 3.12. Let $G$ be a p-group with $Z(G)=C(G) . \quad G$ is critical if and only if $Z(G)$ is cyclic and $G$ is centrally indecomposable.

Proof. Let $G$ be a critical group. Since $G$ is a $p$-group this means $Z(G) \neq 1$. Therefore $C(G)=Z(G) \neq 1$. Hence $G$ is nilpotent of class two. By Theorem 3.1 of [5], (noting that a critical group is equivalent to its not being an in-direct product), $Z(G)$ is cyclic and $G$ may be generated by two elements. Let $G=\{a, b\}$. Since $G$ is not abelian we must have $a, b \notin Z(G)$. Therefore $G / Z(G)$ is of rank two. But $G$ is a $p$-group. It follows that $C(G)$ is a $p$-group. Hence, by Corollary $3.11 G$ is centrally indecomposable.

Conversely, let $Z(G)$ be cyclic and $G$ be centrally indecomposable. By Corollary 3.11, we have $G / Z(G)$ is of rank two. Thus

$$
G=\{a, b, Z(G)\} \text {. }
$$

But $Z(G)=C(G)$. Therefore $G=\{a, b\}$. Hence, by Theorem 3.1 of [5], $G$ must be critical.

Lemma 3.13. Let $G$ be a nilpotent group of class two with a cyclic commutator subgroup. Let $G=\left(\prod_{i=1}^{n} G_{i}\right)_{H}=\left(\prod_{i=1}^{m} F_{i}\right)_{H}$ be two central decompositions of $G$ into centrally indecomposable factors. If $F_{i}=\left\{s_{i}, t_{i}, H\right\}$ such that $\bar{F}_{i}=\left\{\bar{s}_{i}\right\} \times\left\{\bar{t}_{i}\right\}$ then for a given $G_{j}$ there exist subgroups $A_{j} \subset G_{j}$ and $B_{j} \subset G_{j}^{\prime}$ such that $A_{j}=\left\{s_{i}^{\alpha} u_{i}^{-1}, t_{i}^{\alpha} v_{i}^{-1}, H\right\}$ and $B_{j}=\left\{s^{1-\alpha} u_{i}, t_{i}^{1-\alpha} v_{i}, H\right\}$ where $u_{i}, v_{i} \in F_{i}^{\prime}$.

Proof. Consider $\bar{G}=\prod_{i=1}^{n} \bar{G}_{i}=\prod_{i=1}^{m} \bar{F}_{i}$. By Lemma 3.1, there exist $\bar{a} \in \bar{G}_{j}$ and $\bar{b} \in \bar{G}_{j}^{\prime}$ with $\bar{a}=\bar{c} \bar{u}_{i}^{-1}$ and $\bar{b}=\bar{c}^{*} \bar{u}_{i}$ where $\bar{c}, \bar{c}^{*} \in \bar{F}_{i}$ and $\bar{u}_{i} \in \bar{F}_{i}^{\prime}$ such that $\bar{s}_{i}=\bar{a} \bar{b}$. Let $\bar{c}=\bar{s}_{i}^{\alpha} \bar{t}_{i}^{\beta}$ and $\bar{c}^{*}=\bar{s}_{i}^{\varepsilon} \bar{t}_{i}^{\eta}$. This means $\bar{s}_{i}=\bar{s}_{i}^{\alpha+\varepsilon} \bar{t}_{i}^{\beta+\eta}$. Since $\bar{F}_{i}=\left\{\bar{s}_{i}\right\} \times\left\{\bar{t}_{i}\right\}$, it follows that $\bar{t}_{i}^{\eta}=\bar{t}_{i}^{-\beta}$ and $\bar{s}_{i}^{\varepsilon}=\bar{s}_{i}^{1-\alpha}$. Therefore $G_{j}$ contains an element of the form $a=$ $s_{i}^{\alpha} t_{i}^{\beta} u_{i}^{-1}$ and $G_{j}^{\prime}$ contains an element of the form $b=s_{i}^{1-\alpha} t_{i}^{-\beta} u_{i}$ such that 
for a suitable $k \in H$ we have $s_{i}=a b k$. In the same way there exist $a^{*}=s_{i}^{\gamma} t_{i}^{\delta} v_{i}^{-1} \in G_{j}$ and $b^{*}=s_{i}^{-\gamma} t_{i}^{1-\delta} v_{i} \in G_{j}^{\prime}$ such that $t_{i}=a^{*} b^{*} k^{*}$ for some suitable $k^{*} \in H$. Since $\left[G_{j}, G_{j}^{\prime}\right]=\left[F_{i}, F_{i}^{\prime}\right]=1$, therefore, $[a, b]=1$ implies that,

$$
1=\left[u_{i}, u_{i}\right]=\left[s_{i}^{\alpha} t_{i}^{\beta}, s_{i}^{1-\alpha} t_{i}^{-\beta}\right]=h^{-\alpha \beta+(\alpha-1)}=h^{-\beta}
$$

where $h=\left[s_{i}, t_{i}\right]$. Thus $\beta \equiv 0 \bmod q$ where $q=$ ord $h$. In the same way $\left[a^{*}, b^{*}\right]=1$ implies $\gamma \equiv 0 \bmod q$. On the other hand $\left[a, b^{*}\right]=1$ and $\left[a^{*}, b\right]=1$ respectively imply:

$$
\left[u_{i}, v_{i}\right]=\left[s_{i}^{\alpha} t_{i}^{\beta}, s_{i}^{-\gamma} t_{i}^{1-\delta}\right]=h^{\alpha(1-\delta)+\beta \gamma}=h^{\alpha(1-\delta)}
$$

and $\left[u_{i}, v_{i}\right]^{-1}=\left[v_{i}, u_{i}\right]=\left[s_{i}^{\gamma} t_{i}^{\delta}, s_{i}^{1-\alpha} t_{i}^{-\beta}\right]=h^{-\beta \gamma+(\alpha-1) \delta}=h^{(\alpha-1) \delta}$. Therefore $\alpha(1-\delta) \equiv(1-\alpha) \delta \bmod q$, or $\alpha \equiv \delta \bmod q$. Since for any $\lambda$ with $q \mid \lambda$ we have $s_{i}^{\lambda}, t_{i}^{\lambda} \in H$. It follows that $s_{i}^{\alpha} u_{i}^{-1}$ and $t_{i}^{\alpha} v_{i}^{-1}$ are elements of $G_{j}$ and $s_{i}^{1-\alpha} u_{i}$ and $t_{i}^{1-\alpha} v_{i}$ are elements of $G_{j}^{\prime}$. Hence

$$
A_{j}=\left\{s_{i}^{\alpha} u_{i}^{-1}, t_{i}^{\alpha} v_{i}, H\right\} \subset G_{j}
$$

and $B_{j}=\left\{s_{i}^{1-\alpha} u_{i}, t_{i}^{1-\alpha} v_{i}, H\right\} \subset G_{j}^{\prime}$.

THEOREM 3.14. If $G$ is a nilpotent group of class two with a finite cyclic commutator subgroup, then any two central decompositions of $G$ into centrally indecomposable factors are isomorphic $\bmod H$.

Proof. Let $G=\left(\prod_{i=1}^{n} G_{i}\right)_{H}=\left(\prod_{i=1}^{m} F_{i}\right)_{H}$ be two central decompositions of $G$ into centrally indecomposable factors. Since $C(G)$ is cyclic, by Theorem 3.9, $\bar{G}_{i}$ and $\bar{F}_{i}$ are abelian of rank two for all $i$. In particular we shall let $F_{i}=\left\{s_{i}, t_{i}, H\right\}$ such that $\bar{F}_{i}=\left\{s_{s}\right\} \times\left\{t_{i}\right\}$. Thus, by Lemma 3.13 , and using the same notations, there exist $A_{j} \subset G_{j}$ and $B_{j} \subset G_{j}^{\prime}$. Since $C(G)$ is finite and cyclic, by Theorem 3.8, $C\left(F_{i}\right)$ must be a cyclic $p$-group. Let $p^{\lambda}=$ ord $C\left(F_{i}\right)$. Then either $\alpha$ or $(1-\alpha)$ must be relatively prime to $p$. Therefore either $\left\{s_{i}^{\alpha}, t_{i}^{\alpha}, H\right)$ or $\left\{s_{i}^{1-\alpha}, t_{1}^{1-\alpha}, H\right\}$ must give the group $F_{i}$.

CASE 1. $F_{i}=\left\{s_{i}^{\alpha}, t_{i}^{\alpha}, H\right\}$ for all $j$. (Note: $\alpha, u_{i}, v_{i}$ are dependent on $G_{j}$ ) In particular we shall let $i=j$. Since $G_{i}$ is centrally indecomposable, therefore, by Lemma $3.4, G_{i}=A_{i}$. A simple check will show that $F_{i}$ and $\bar{G}_{i}$ are exchangeable in the decompositions

$$
\bar{G}=\prod_{\nu=1}^{n} \bar{G}_{\nu}=\prod_{\nu=1}^{m} \bar{F}_{\nu} .
$$

Thus by Lemma 2.2, $\bar{F}_{i} \simeq \bar{G}_{i}$. Moreover, applying Lemma 2.3, we must have $m=n$. Hence the two central decompositions are isomorphic $\bmod H$. 
CASE 2. There exists $j$ such that $F_{i}=\left\{s_{i}^{1-\alpha}, t_{i}^{1-\alpha}, H\right\}$. We shall prove this case by induction on $n$. Clearly the theorem is true for $n=1$. Consider $G=\left(G_{j} \times G_{j}^{\prime}\right)_{H}=\left(F_{i} \times F_{i}^{\prime}\right)_{H}$. Since $C\left(F_{i}\right)$ is a cyclic $p$-group therefore ord $\left[s_{i}, t_{i}\right]$ must be a power of $p$. Moreover

$$
\left[u_{i}, v_{i}\right] \in\left\{\left[s_{i}, t_{i}\right]\right\} \text {. }
$$

Therefore $C\left(B_{j}\right)$ must be a cyclic p-group. Hence by Theorem 3.8 $B_{j}$ is centrally indecomposable. Moreover, by Lemma 3.4,

$$
G_{j}^{\prime}=\left(B_{j} \times N\right)_{H}
$$

where $N=G_{j}^{\prime} \cap F_{i}^{\prime}$. Therefore, by induction, $\bar{B} \simeq \bar{G}_{k}$ for some $k \neq j$ and any central decompositions of $N$ into centrally indecomposable factors are isomorphic $\bmod H$ to $\left(\prod_{\nu \neq k j}^{n} G_{\nu}\right)_{H}$. A simple check will show that in the decompositions $\bar{G}=\bar{G}_{j} \times \bar{B}_{j} \times \bar{N}=\bar{F}_{i} \times \bar{F}_{i}^{\prime}, \bar{B}_{j}$ and $\bar{F}_{i}$ are exchangeable. Thus $\bar{B}_{j} \simeq \bar{F}_{i}$. Furthermore by the construction of $A_{j}$ and $B_{j}$ we have $F_{i} \subset\left\{A_{j}, B_{j}\right\}$. Therefore $F_{i} \subset\left(G_{j} \times B_{j}\right)_{H}$. Let $U=F_{i}^{\prime} \cap\left(G_{j} \times B_{j}\right)_{H}$. We shall show that $P=\left(G_{j} \times B_{j}\right)_{H}=$ $\left(F_{i} \times U\right)_{H}$. It is clear that $\left[F_{i}, U\right]=1$ and $F_{i} \cap U=H$. Let

$$
x \in\left(G_{j} \times B_{j}\right)_{H} \text {. }
$$

Then $x=g b$ for sme $g \in G_{j}$ and $b \in B_{j}$. But $g=f u$ and $b=f^{*} u^{*}$ for some $f, f^{*} \in F_{i}$ and $u, u^{*} \in F_{i}^{\prime}$. Since $F_{i} \subset P$ we must have $u, u^{*} \in P$. Hence $x=\left(f f^{*}\right)\left(u u^{*}\right)$ where $f f^{*} \in F_{i}$ and $u u^{*} \in\left(F_{i}^{\prime} \cap P\right)=U$. Thus we have,

$$
G=\left(G_{j} \times B_{j} \times N\right)_{H}=\left(F_{i} \times U \times N\right)_{H}=\left(F_{i} \times F_{i}^{\prime}\right)_{H} .
$$

Since $(U \times N)_{H} \subset F_{i}^{\prime}$ we must have $(U \times N)_{H}=F_{i}^{\prime}$. We must now show that $U$ is centrally indecomposable. Since $G_{j}$ is centrally indecomposable there exist $g, g^{*} \in G_{j}$ such that $G_{j}=\left\{g, g^{*}, H\right\}$ and $\bar{G}_{j}=$ $\{\bar{g}\} \times\left\{\bar{g}^{*}\right\}$. Let $g=f u$ and $g^{*}=f^{*} u^{*}$, where $f, f^{*} \in F_{i}$ and $u, u^{*} \in F_{i}^{\prime}$. Thus if $x \in G_{j}$ and $x=y v$ where $y \in F_{i}$ and $v \in F_{i}^{\prime}$ we have,

$$
x=y v=w\left(g, g^{*}\right) h=w\left(f, f^{*}\right) \cdot w\left(u, u^{*}\right) \cdot h
$$

where $h \in H$ and $w\left(g, g^{*}\right)$ is a word on $g, g^{*}$ (we shall regard $w$ as a function). Since $y$ and $w\left(f, f^{*}\right)$ are elements of $F_{i}$, therefore, $v \in$ $\left\{u, u^{*}, H\right\}$. In particular we note that $u_{i}$ and $v_{i}$ are elements of $\left\{u, u^{*}, H\right\}$. Since $F_{i} \subset P$ it is not difficult to see that $\left\{u, u^{*}, H\right\} \subset P$ and indeed $U=\left\{u, u^{*}, H\right\}$. Furthermore $C\left(G_{j}\right)$ is a cyclic p-group. Therefore $C(U)$ must be a cyclic p-group. Hence $U$ is centrally indecomposable. Recalling that any central decomposition of $N$ into centrally indecomposable factors is isomorphic $\bmod H$ to $\left(\prod_{\nu \neq j, k}^{n} G_{\nu}\right)_{H}$ it follows that in the central decomposition of $F_{i}^{\prime}=(U \times N)_{H}$ into indecomposable factors there will be exactly $(n-1)$ indecomposable 
factors. Hence by induction $F_{i}^{\prime}=\left(\prod_{\nu \neq i}^{m} F_{\nu}\right)_{H}$ is isomorphic $\bmod H$ to $F_{i}^{\prime}=\left(U \times \prod_{\nu=1}^{n-2} N_{\nu}\right)_{H}$, where $N=\left(\prod_{\nu=1}^{n-2} N_{\nu}\right)_{H}$ and $N_{\nu}$ centrally indecomposable and $m-1=n-1$.

We now note that in the decompositions of $\bar{P}=\left(\bar{G}_{j} \times \bar{B}_{j}\right)=$ $\left(\bar{F}_{i} \times \bar{U}\right), \bar{B}_{j}$ and $\bar{F}_{i}$ are exchangeable. This implies $\bar{G}_{j}$ and $\bar{U}$ are exchangeable in these two decompositions. Thus $\bar{G}_{j}$ and $\bar{U}$ are isomorphic. Hence with suitable re-indexing we can have $\bar{G}_{\lambda} \simeq \bar{F}_{\lambda}$ where $G_{\lambda}$ and $F_{\lambda}$ are re-indexed factors of $G_{k}^{\prime}$ and $F_{i}^{\prime}$ respectively. Recalling that $\bar{G}_{k} \simeq \bar{B}_{j}$ and $\bar{B}_{j} \simeq \bar{F}_{i}$ the theorem follows immediately.

The author wishes to thank the referee for his suggestions.

\section{REFERENCES}

1. B. H. Neumann and H. Neumann, A remark on generalised free products, J. London Math. Soc. 25 (1950), 202-204.

2. H. Neumann, Generalised free sums of cyclical groups, Amer. J. Math. 72 (1950), 671-685.

3. - On an amalgam of abelian groups, J. London Math. Soc. 26 (1951), 228232.

4. C. Y. Tang, An existence theorem for generalized direct products with amalgamated subgroups, Canad. J. Math. 18 (1966), 75-82.

5. P. M. Weichsel, A decomposition theory for finite groups with applications to $p$ groups, Trans. Amer. Math. Soc. 102 (1962) 218-226.

6. H. Zassenhaus, The theory of groups, Chelsea, New York, 1958.

Received July 30, 1966.

UNIVERSITY OF WATERLOO

ONTARIO, CANADA 


\section{PACIFIC JOURNAL OF MATHEMATICS}

\section{EDITORS}

\section{H. ROYDEN}

Stanford University

Stanford, California

J. P. JANS

University of Washington

Seattle, Washington 98105

\section{J. DUGUNDJI}

Department of Mathematics

Rice University

Houston, Texas 77001

RICHARD ARENS

University of California

Los Angeles, California 90024

ASSOCIATE EDITORS

E. F. BECKENBACH

B. H. NeumanN

F. WoLF

K. YosidA

\section{SUPPORTING INSTITUTIONS}

\author{
UNIVERSITY OF BRITISH COLUMBIA \\ CALIFORNIA INSTITUTE OF TECHNOLOGY \\ UNIVERSITY OF CALIFORNIA \\ MONTANA STATE UNIVERSITY \\ UNIVERSITY OF NEVADA \\ NEW MEXICO STATE UNIVERSITY \\ OREGON STATE UNIVERSITY \\ UNIVERSITY OF OREGON \\ OSAKA UNIVERSITY \\ UNIVERSITY OF SOUTHERN CALIFORNIA
}

\author{
STANFORD UNIVERSITY \\ UNIVERSITY OF TOKYO \\ UNIVERSITY OF UTAH \\ WASHINGTON STATE UNIVERSITY \\ UNIVERSITY OF WASHINGTON \\ AMERICAN MATHEMATICAL SOCIETY \\ CHEVRON RESEARCH CORPORATION \\ TRW SYSTEMS \\ NAVAL ORDNANCE TEST STATION
}




\section{Pacific Journal of Mathematics}

\section{Vol. 23, No. 1 \\ March, 1967}

M. J. C. Baker, A spherical Helly-type theorem ................... 1

Robert Morgan Brooks, On locally m-convex*-algebras.............. 5

Lindsay Nathan Childs and Frank Rimi DeMeyer, On automorphisms of separable algebras ...................................

Charles L. Fefferman, A Radon-Nikodym theorem for finitely additive set

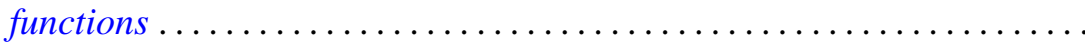

Magnus Giertz, On generalized elements with respect to linear

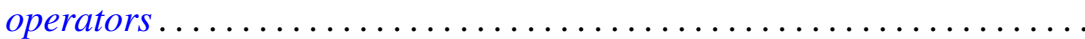

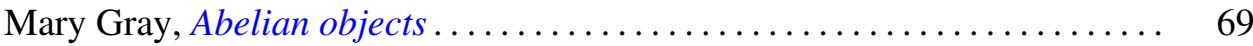

Mary Gray, Radical subcategories.............................. 79

John A. Hildebrant, On uniquely divisible semigroups on the two-cell . . . . . 91

Barry E. Johnson, AW*-algebras are $\mathrm{QW}^{*}$-algebras ............... 97

Carl W. Kohls, Decomposition spectra of rings of continuous functions . . . . 101

Calvin T. Long, Addition theorems for sets of integers .............. 107

Ralph David McWilliams, On $w^{*}$-sequential convergence and quasi-reflexivity ................................... 113

Alfred Richard Mitchell and Roger W. Mitchell, Disjoint basic

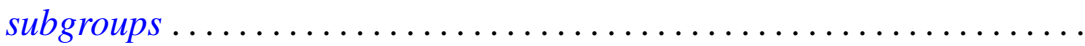

John Emanuel de Pillis, Linear transformations which preserve hermitian and positive semidefinite operators .

Qazi Ibadur Rahman and Q. G. Mohammad, Remarks on Schwarz's lemma

Neal Jules Rothman, An $L^{1}$ algebra for certain locally compact topological semigroups ...

F. Dennis Sentilles, Kernel representations of operators and their adjoints ...

D. R. Smart, Fixed points in a class of sets

K. Srinivasacharyulu, Topology of some Kähler manifolds

Francis C.Y. Tang, On uniqueness of generalized direct decompositions .

171 Albert Chapman Vosburg, On the relationship between Hausdorff dimension and metric dimension . 